\title{
10
}

\section{DATA FUSION VIA FISSION FOR THE ANALYSIS OF BRAIN DEATH}

L. Li, Y. Saito, D. Looney, T. Tanaka, J. Cao, D. Mandic

\begin{abstract}
Information fusion via signal fission is addressed in the framework of empirical mode decomposition (EMD) to determine brain death in deep coma patients. In this way, a general nonlinear and nonstationary brain signal is decomposed into its oscillatory components (fission); the components of interest are then combined in an ad-hoc or automated fashion in order to provide greater knowledge about a process in hand (fusion). This chapter illustrates how the fusion via fission methodology can be used to retain components of interest in electroencephalography (EEG), thus highlighting the absence or presence of brain death. Additionally, it is shown how complex extensions of the algorithm can be used to detect phase synchronization by simulations and applications to EEG signals.
\end{abstract}

\subsection{INTRODUCTION}

As modern society evolves, there is an ever-growing requirement for more focused information rather than superfluous data. The ability to take measurements and gather data is generally well developed. But optimizing data gathering and information production, which is vital for economic and social progress, are in their infancy. For example, it is difficult via conventional means to characterize signals that display highly

Evolving Intelligent Systems: Methodology and Applications, Edited by Plamen Angelov, Dimitar P. Filev, and Nikola Kasabov

Copyright (C) 2010 Institute of Electrical and Electronics Engineers 
complex nonlinear and nonstationary behavior caused by either the measurement equipment or the interconnection of data from different sources.

Data analysis methods, which are not limited to linear, deterministic, or stationary signals, are needed for the analysis of real-world data. Recently, information fusion via fission has been realized [15] by decomposing input signals into a set of their components using empirical mode decomposition (EMD) (originally introduced in [10]) and then fusing these components using suitable criteria. In this chapter, we apply this methodology to the area of analyzing electroencephalogram (EEG) data recorded from the brain. We illustrate, through experimental results, how the characteristics of the underlying data can be extracted via EMD.

\subsection{DATA FUSION VIA FISSION}

Time-domain analysis illustrates amplitude changes with time. However, by examining data in the frequency domain, it is often possible to extract more useful information, especially when examining signals obtained from the brain. Fourier-based analysis is the traditional data analysis method and regarded as the best-established tool for the processing of linear and stationary data. In the real world, data are nonlinear and nonstationary, for which Fourier analysis is not well suited, it is required to resort to time-frequency analysis techniques such as the short timefourier transform (STFT) and wavelet transform $(W T)$. Despite the power of these techniques, they still rely on some kind of projection on a set of predefined bases; this makes some areas of their application, particularly when focusing on high-frequency content, rather limited [10]. Therefore, empirical mode decomposition [10] was first introduced as a fully data-driven technique that makes no assumptions on the underlying data being analyzing. It decomposes the signal into a set of zero mean components, which are called intrinsic mode functions (IMFs).

EMD has enormous appeal for nonlinear and nonstationary signals because it makes no prior assumptions about the data and, as such, it belongs to the class of exploratory data analysis (EDA) techniques [20]. The original algorithm was successfully applied to a number of problems that require high resolution but are separable in the time-frequency domain, such as in ocean engineering [6], biomedical signal processing [18] and seismics [23]. Although EMD has primarily been used for time-frequency analysis, it is clear that the IMFs represent the oscillation modes embedded in the data and therefore has potential within the framework of data fusion and feature extraction $[12,13]$.

Data and information fusion is the approach whereby data from multiple sensors or components are combined to achieve improved accuracies and more specific inferences that could not be achieved by the use of only a single sensor [9]. Its principles have been employed in a number of research fields, including information theory, signal processing, and computing [7, 9, 21, 22]. Recent work [14] demonstrates that the decomposition nature of EMD provides a unifying framework for information fusion via fission, where fission is the phenomenon by which observed information is decomposed into a set of its components. More specifically, the stages of signal processing, feature extraction, and situation assessment from the waterfall model (a well-established fusion model given in Figure 10.1) can all be achieved by EMD. 


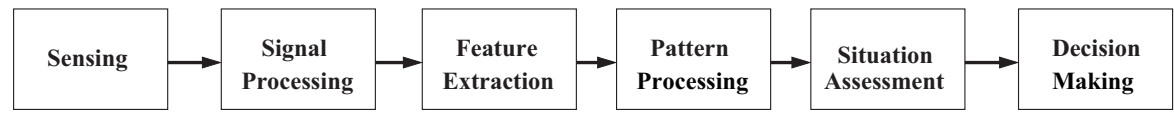

Figure 10.1. Waterfall model of information fusion.

A simple example of the fission and feature extraction properties of EMD is its ability to separate original input components from a signal composed of linear sinusoids, as shown in Figure 10.2. The signal "U" shown at the top of the figure is a linear sum of three sinusoids, with individual frequencies of $0.1 \mathrm{~Hz}, 1 \mathrm{~Hz}$, and $10 \mathrm{~Hz}$, represented below as:

$$
U(t)=\sin (0.2 \pi t)+\sin (2 \pi t)+\sin (20 \pi t)
$$

The above signal is decomposed into its IMFs with EMD. The IMFs are denoted by $C_{i}(\mathrm{k}), i=1,2, \ldots, \mathrm{M}$ in the subsequent panels on Figure 10.2. Note that the IMFs represent the original sinusoidal components used to create U. Most importantly, though, EMD has distinct advantages over other decomposition techniques in analyzing real-world signals as it does not make unrealistic assumptions about the data.
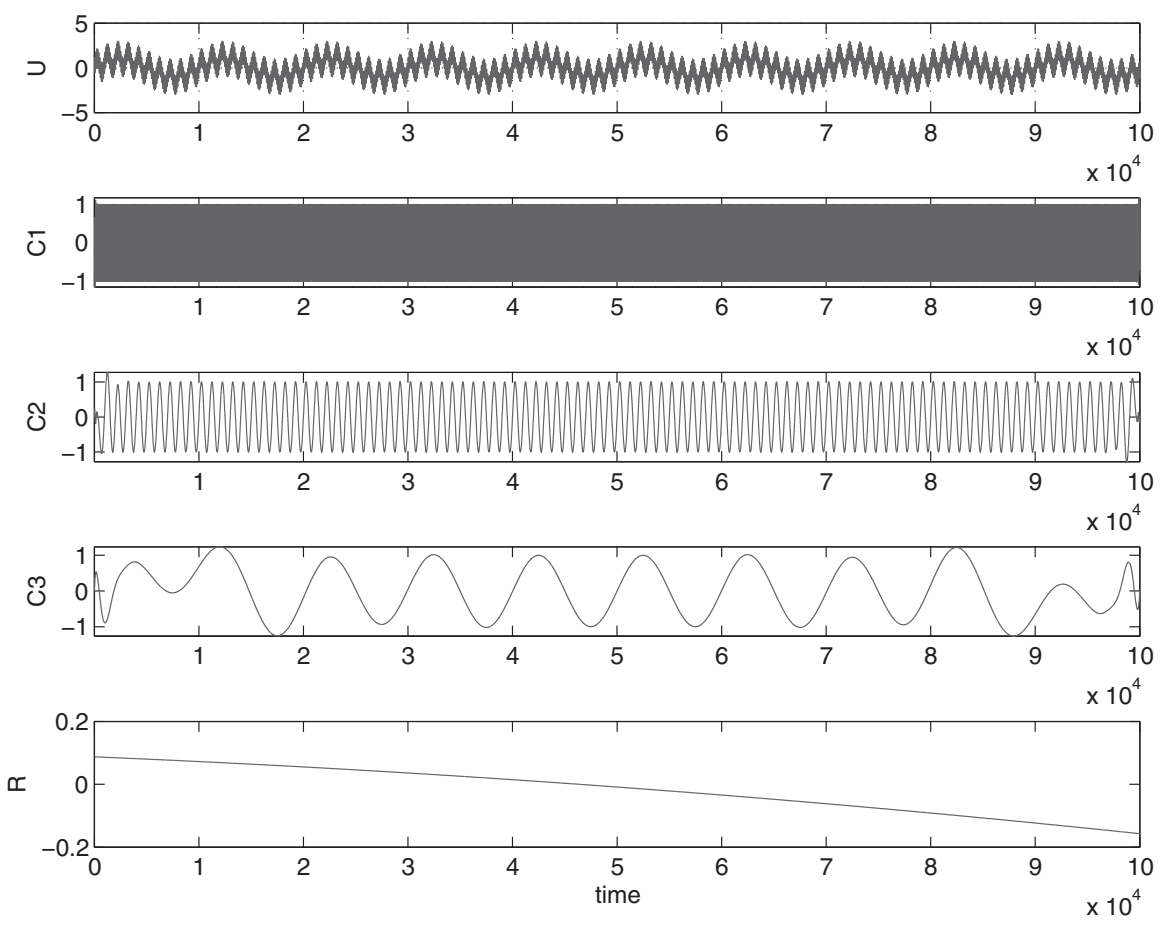

Figure 10.2. Example of data fission via EMD. 
Principal component analysis (PCA), for example, assumes linearity and stationarity. As a result, PCA performs poorly when processing real-world signals that display nonlinear and nonstationary behavior such as biomedical data [18]. Similar criticisms can be made of independent component analysis (ICA), which requires that input components be statistically independent. This issue is highlighted by the ability of EMD to decouple noisy recordings in the problem of blood volume estimation [18].

\subsection{BRAIN DEATH DETECTION}

The investigation of the brain's consciousness, especially the state of brain death, is highly important. Brain death is currently used as a definition for death among the legal and medical communities. It is for this reason that brain death needs to be determined precisely so as not to cause patients potential risk.

The legal definition of brain death is an irreversible loss of forebrain and brainstem functions [2]; however, it is very difficult to realize brain death diagnosis precisely due to clinical difficulties. Specialized personnel and technology are needed to perform a series of tests that are expensive and time consuming and can sometimes bring risk to the patient. In this chapter, brain death means an initial diagnosis.

Some of the tests require that medical care instruments be removed. Further, some tests require that the patient be transported out of the intensive care unit (ICU) and others (confirmatory test) need to be performed several times at an interval of up to 10 hours and can take as long as 30 minutes. After initial documentation of the clinical signs of brain death, repetition of clinical testing is required by a number of countries. Although the diagnosis criteria are different from country to country, these tests can bring the patient a potential medical risk of less medical care due to the requirements of implementing test and stress to the already compromised organ [16].

To overcome these difficulties, a preliminary EEG test has been proposed [3] to determine whether further brain death tests, especially those requiring patients to be disconnected from important medical care (the respirator), need to be implemented. This is clearly of great benefit as it reduces potential complications and stress for patients. The test is not trivial, however, as interference to the EEG signal is caused by various other medical instruments in the ICU. As a result, these interferences can contaminate the EEG signal we want to investigate. In the past, ICA has been used to perform the preliminary test [3]. But, for reasons explored earlier, ICA is limited by its unrealistic assumptions. Therefore, data fusion via fission through empirical mode decomposition is introduced in this chapter to analyze EEG signals and extract useful information without making assumptions of the input EEG signal.

\subsection{EEG DATA}

\subsubsection{Introduction}

Electroencephalography $(E E G)$ is regarded as a vital clinical monitoring tool in understanding the brain activity by recording the voltage differences between different 
parts of brain using electrodes placed on the scalp, subdurally or in the cerebral cortex. Essentially, EEG can detect brain activities by highlighting the voltage differences generated by neurons in the brain.

\subsubsection{EEG Data Acquisition}

The standardized 10-20 system for collecting EEG data was originally described by Jasper in 1958 [11] for placement of electrodes. There are also other systems such as the 10-10 system, an extension to the original 10-20 system with a higher channel density of 81, suggested by [4]. But the 10-20 system has also been accepted as the standard of the American Clinical Neurophysiology Society (formerly the American Electroencephalographic Society) and the International Federation of Clinical Neurophysiology (formerly the International Federation of Societies for Electroencephalography and Clinical Neurophysiology). The 10-20 system was used to collect the EEG data for our experiments.

In our analysis of brain death, the patients involved were all lying in bed facing up with eyes closed, and the data was obtained via nine electrodes on the forehead with channels based on the 10-20 system. That is, electrodes were placed at positions F3, F4, F7, F8, Fp1, Fp2, as well as GND, and also two were placed on the ears (denoted by A1 and A2 respectively). The electrodes placed on the ears act as a reference for the measurements, which can be calculated as $(\mathrm{A} 1+\mathrm{A} 2) / 2$. The measured voltage signal is then digitalized via a portable EEG recording instrument with a sampling frequency of $1000 \mathrm{~Hz}$. The position of the electrodes and its output signal can be seen in Figure 10.3.

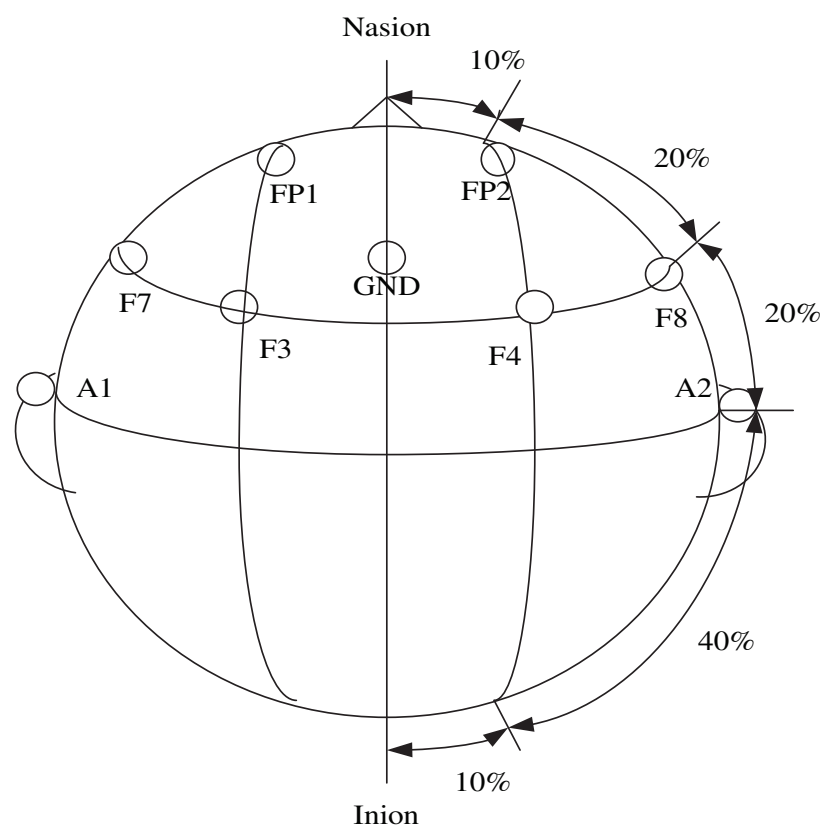

Figure 10.3. Electrode placement. 
TA B LE 10.1. Experimental Data

\begin{tabular}{lccccr}
\hline Type of Patient & Coma & Quasi-Brain-Death & Male & Female & Total \\
\hline Number of Patients & 19 & 18 & 20 & 16 & 36 \\
\hline
\end{tabular}

\subsubsection{Patients' Data}

The experimental data shown in Table 10.1 was obtained from 36 patients (16 female, 20 male) of ages ranging from 17 to 85 years old from 2004 until 2006. Nineteen of the patients were in a state of coma, 18 of them need a further test to determine its status, and the consciousness of one patient was clinically in a state of uncertainty. Total recordings of EEG signals from these 36 patients with an average signal length of five minutes were stored and analyzed.

To illustrate, two sets of EEG signals are shown here: Figure 10.4 is the EEG signal of patient 1: a 64-year-old male patient in a deep coma state. The patient regained consciousness. Figure 10.5 is the EEG signal of patient 2: a 48 -year-old male patient who lost consciousness on October 18, 2005.

The signal shown in Figure 10.4 is the time-amplitude plot of the EEG signal from patient 2 in a deep coma status. In the figure, from top to bottom, the six EEG channels are shown individually with the same time slot of 40,000 seconds. Figure 10.5 presents the EEG signal of the same patient in a brain-death status.

\subsubsection{EEG Frequency Band and Its Relative Behavior}

EEG signals are typically nonlinear and nonstationary data that are composed of frequency components of interest up to $40 \mathrm{~Hz}$. The basic EEG frequencies are summarized briefly in Table 10.2, with regard to their typical distribution on the scalp and subject states [5]. The EEG signal is closely related to the level of consciousness of the person. As the brain activity increases, the higher frequency bands of the EEG become more dominant.

\subsection{EMPIRICAL MODE DECOMPOSITION}

\subsubsection{The EMD Algorithm}

The empirical mode decomposition and Hilbert-Huang transform (HHT) were introduced specifically for analyzing data from nonlinear and nonstationary processes by Huang et al. [10]. The EMD method adaptively decomposes the signals into a set of zeromean components. As these components, called intrinsic mode functions (IMFs), are band-limited signals, the Hilbert transform can be applied to determine a unique time-frequency representation of the data. The decomposition of a signal into a set of IMFs is achieved using the sifting algorithm: 


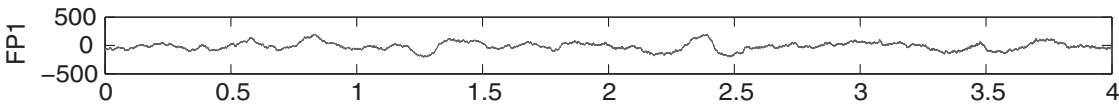

$\times 10^{4}$

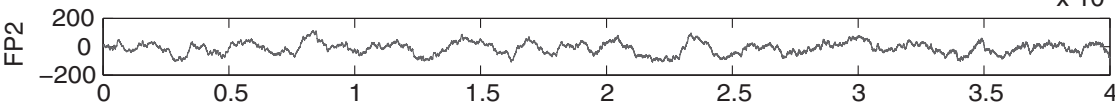
$\times 10^{4}$

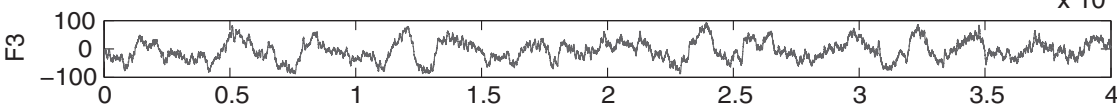
$\times 10^{4}$

吉

I4

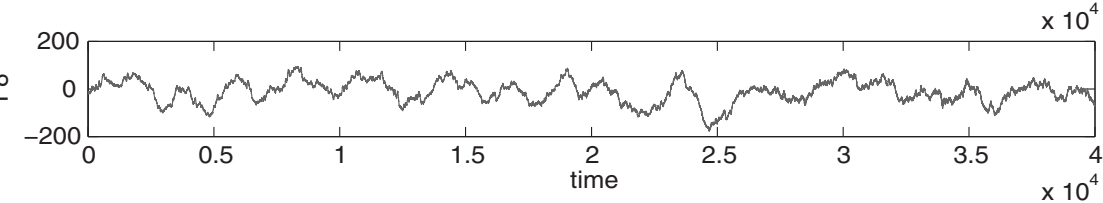

Figure 10.4. Coma signal.

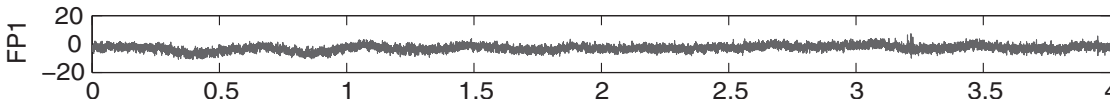

$\times 10^{4}$

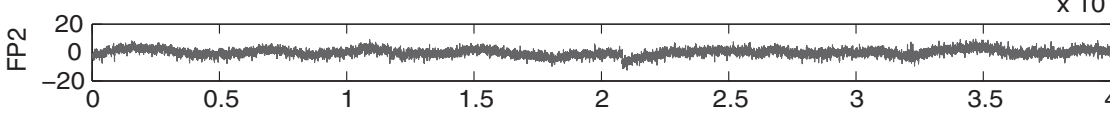

$\times 10^{4}$

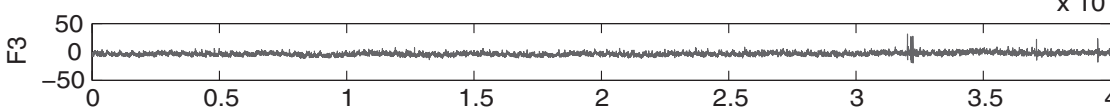

$\times 10^{4}$

苀 $\begin{array}{r}20 \\ 0\end{array}$ $\times 10^{4}$
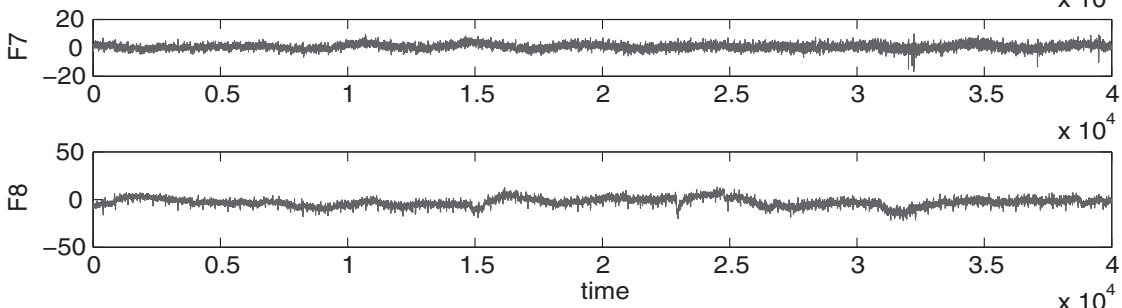

Figure 10.5. Brain death signal. 
TA B LE 10.2. EEG Signal Frequency Band

\begin{tabular}{|c|c|c|c|}
\hline $\begin{array}{l}\text { Frequency } \\
\text { Band }\end{array}$ & $\begin{array}{l}\text { Frequency } \\
\qquad(\mathrm{Hz})\end{array}$ & Consciousness Level & Distribution \\
\hline Delta & $0.5-4$ & Low level of arousal & Generally broad, diffused \\
\hline Theta & $4-8$ & Distracted & Regional, involves many lobes \\
\hline Alpha & $8-12$ & Relaxed, meditation & Regional, involves entire lobe \\
\hline Low Beta & $12-15$ & Relaxed, yet focused & $\begin{array}{l}\text { By side and lobe (frontal, } \\
\text { occipital, etc.) }\end{array}$ \\
\hline Midrange Beta & $15-18$ & Alert, active, not agitated & Localized, over various areas \\
\hline High Beta & $>18$ & Very focused, alertness & $\begin{array}{l}\text { Very localized, maybe very } \\
\text { focused }\end{array}$ \\
\hline Gamma & 40 & High-level info processing & Very localized \\
\hline
\end{tabular}

\section{Sifting Algorithm}

1. Find the local maxima and connect the maxima with interpolation (max envelope). Also extract the minima and connect them by interpolation (min envelope).

2. Take the average of the max envelope and the min envelope to determine the signal mean.

3. Subtract the signal mean obtained from step 2 from the original signal.

4. Repeat steps 1, 2, and 3 until the signal satisfies the IMF criteria defined by Huang [10].

5. Subtract the IMF signal from the original signal; a new signal is then obtained. Again, steps 1, 2, 3, 4, and 5 should be implemented to extract IMFs until the residual is a monotonic function.

The decomposition of a signal $\mathrm{x}(\mathrm{t})$ into IMFs can be summarized by the following equation:

$$
x(t)=\sum_{i=1}^{n}\left(C_{i}(t)\right)+r(t)
$$

where $C_{i}(t)$ is the $i$ th IMF, $n$ is the total number of IMFs, and $r(t)$ is the residue.

The criteria of an IMF are as follows. The first condition is that the number of local extrema and the number of zero crossings must either be equal or differ at most by one. The second condition is that at any point the mean of the signal defined by the local maxima and the local minima is zero.

\subsubsection{The Hilbert Spectrum and Instantaneous Frequency}

The monocomponent nature of the IMFs facilitates a unique time-frequency representation of the original signal-the Hilbert-Huang spectrum. The Hilbert transform is 
given by [8]:

$$
\tilde{x}(t)=\mathcal{H}[x(t)]=\int_{-\infty}^{\infty} \frac{x(u)}{\pi(t-u)} d u
$$

where $x(t)$ is a time domain signal. $\mathcal{H}[\cdot]$ is the Hilbert operator. The analytic signal $z(t)$ is related to the Hilbert transform of $x(t)$, represented below as:

$$
z(t)=x(t)+j \tilde{x}(t)
$$

Given the analytic representation, the magnitude function $a(t)$ and phase function $\theta(t)$ can be obtained from $z(t)$ as:

$$
\begin{gathered}
a(t)=\sqrt{x^{2}(t)+\tilde{x}^{2}(t)} \text { and } \theta(t)=\arctan \left(\frac{\tilde{x}(t)}{x(t)}\right) \\
z(t)=a(t) e^{j \theta(t)}
\end{gathered}
$$

where $a(t)$ describes the envelope of the original signal $x(t)$, in the other words, the instantaneous amplitude of the signal [8]. $\theta(t)$ describes the instantaneous phase of $x(t)$. Therefore, the instantaneous frequency of $x(t)$ can be defined as the derivative of the phase of the signal as:

$$
\omega(t)=\frac{d}{d t} \theta(t)
$$

Thus, the Hilbert spectrum is given by [10]:

$$
x(t)=\sum_{i=1}^{n} a_{i}(t) \exp \left(j \int \omega_{i}(t)\right) d t
$$

Given the above representation equation, a three-dimensional plot with the amplitude, instantaneous frequency as a function of time can be obtained in a straightforward fashion. This is the Hilbert spectrum.

\subsubsection{Importance of Complex EMD}

The extension to the complex domain $\mathbb{C}$ is particularly important for the analysis of phasedependent processes [15]. This applies not only to naturally occurring complex data, but also to multi-channel real data. In this way, it is possible to analyze mutual phase information between real-valued signals.

The first method of employing EMD in the complex domain, complex empirical mode decomposition, was introduced in 2007 [19]. The method is rigorously derived, based on the inherent relationship between the positive and negative frequency components of a complex signal and the properties of the Hilbert transform. The idea behind this approach is rather intuitive: first note that a complex signal has a two-sided, asymmetric 
spectrum. The complex signal can therefore be converted into a sum of analytic signals by a straightforward filtering operation that extracts the opposite sides of the spectrum. Direct analysis in $\mathbb{C}$ can be subsequently achieved by applying standard EMD to both the positive and negative frequency parts of the signal. Given a complex signal $x(k)$, realvalued components corresponding to the positive and negative sides of the spectrum can be extracted as

$$
\begin{aligned}
& x_{+}(k)=\mathcal{R} \mathcal{F}^{-1}\left\{X\left(e^{j \omega}\right) \cdot H\left(e^{j \omega}\right)\right\} \\
& x_{-}(k)=\mathcal{R} \mathcal{F}^{-1}\left\{X\left(e^{j \omega}\right) \cdot H\left(e^{-j \omega}\right)\right\}
\end{aligned}
$$

where $\mathcal{F}^{-1}$ is the inverse Fourier transform, $\mathcal{R}$ is an operator that extracts the real part of a signal, and $H\left(e^{j \omega}\right)$ is an ideal filter with the transfer function

$$
H\left(e^{j \omega}\right)= \begin{cases}1, & \omega>0 \\ 0, & \omega<0\end{cases}
$$

Given that $x_{+}(k)$ and $x_{-}(k)$ are real valued, standard EMD can be applied to obtain a set of IMFs for each analytic signal. This can be expressed as

$$
\begin{aligned}
& x_{+}(k)=\sum_{i=1}^{M_{+}} x_{i}(k)+r_{+}(k) \\
& x_{-}(k)=\sum_{i=-M_{-}}^{-1} x_{i}(k)+r_{-}(k)
\end{aligned}
$$

where $\left\{x_{i}(k)\right\}_{i=1}^{N_{+}}$and $\left\{x_{i}(k)\right\}_{i=-N_{-}}^{i=1}$ denote sets of IMFs corresponding, respectively, to $x_{+}(k)$ and $x_{-}(k)$, whereas $r_{+}(k)$ and $r_{-}(k)$ are the respective residuals. The original complex signal can be reconstructed by

$$
x\left[(k)=\left(x_{+}(k)+j \mathcal{H}\left[x_{+}(k)\right]\right)+\left(x_{-}(k)+j \mathcal{H}\left[x_{-}(k)\right]\right)^{*}\right.
$$

where $\mathcal{H}$ is the Hilbert transform operator. To conclude the derivation, a single set of complex IMFs corresponding to the complex signal $x(k)$ is given by

$$
x(k)=\sum_{i=M_{-}, i \neq 0}^{M_{+}} y_{i}(k)+r(k)
$$

where $r(k)$ is the residual and $y_{i}(k)$ is defined by

$$
y_{i}(k)= \begin{cases}\left(x_{+}(k)+j \mathcal{H}\left[x_{+}(k)\right]\right), & i=1, \ldots, M_{+}, \\ \left(x_{-}(k)+j \mathcal{H}\left[x_{-}(k)\right]\right)^{*}, & i=-M_{-}, \ldots,-1\end{cases}
$$

Although the two time series $\left(x_{1}(k)\right.$ and $\left.x_{2}(k)\right)$ are real valued, a complex signal, $z(k)=x_{1}(k)+j x_{2}(k)$, is constructed with the signals representing the real and imaginary parts, respectively. To its advantage, it has a straightforward, intuitive mathematical 
derivation. As an example of its mathematical robustness, it preserves the dyadic filter property of standard EMD. In other words, the algorithm acts as dyadic filter bank when processing complex noise [19].

Rotation-invariant EMD, introduced in [1], proposes a way of extending EMD theory so that it operates fully in $\mathbb{C}$. This is achieved through the use of complex splines. Unlike complex EMD, by design this method generates an equal number of IMFs for the real and imaginary parts; these can be given physical meaning, thus retaining an important property of real-valued EMD. A critical aspect of the derivation of EMD in $\mathbb{C}$ is the definition of an extremum. Several possible approaches are suggested in [1], such as the extrema of the modulus and the locus where the angle of the first-order derivative (with respect to time) changes sign; this way it can be assumed that local maxima will be followed by local minima (and vice versa). This definition is equivalent to the extrema of the imaginary part, that is, for a complex signal $Z(t)$ (for convenience we here use a continuous time index $t$ ):

$$
\begin{aligned}
\angle \dot{Z}(t) & =0 \Rightarrow \angle\{\dot{x}(t)+j \cdot \dot{y}(t)\}=0 \\
\Rightarrow \tan ^{-1} & \frac{\dot{y}(t)}{\dot{x}(t)}=0 \Rightarrow \dot{y}(t)=0
\end{aligned}
$$

Because of its aforementioned advantages over complex EMD, namely the physical meaning of its IMFs, rotation-invariant EMD is the extension of choice to perform analysis on complex data.

\subsubsection{Experiments and Results}

As stated previously, an EEG signal can reflect brain activities; therefore, it is regarded as a powerful tool to analyze cerebral consciousness status (e.g., coma and quasi-braindeath). We distinguish cerebral consciousness status by analyzing EEG frequency components within the frequency range from $0 \mathrm{~Hz}$ to $30 \mathrm{~Hz}$; in particular, we are interested (See Table 10.2) in the delta range $(0.5$ to $4 \mathrm{~Hz})$, theta range (4 to $8 \mathrm{~Hz}$ ), alpha wave ( 8 to $12 \mathrm{~Hz}$ ), and $13 \mathrm{~Hz}$ components within the low beta range. This is because the patients are in a state of coma, as noted before, and this means that the brain activity of interest dominates lower-frequency ranges.

We consider two patients (patient 1 and patient 2) in a coma state. EEG data for each patient has been provided by [3]. Since these recordings were collected, patient 1 has a wakened from coma while patient 2 has died. It is proposed to apply EMD to extract the relevant features from the EEG data and to illustrate how these features can be used to detect inevitable brain death in patient 2. Time-amplitude plots of length one second for each of the six EEG channels (FP1, FP2, . , etc.) for patient 1 and patient 2 are given in the left columns of Figures 10.6 and 10.7, respectively. EMD was applied to each of the channels to decompose them into their IMFs and the resulting Hilbert spectrum was computed. The Hilbert spectrum of each channel within the range 0 to $30 \mathrm{~Hz}$ is given in Figures 10.6 and 10.7, respectively. The third column of each figure shows the frequency-amplitude plots for each channel obtained by averaging the corresponding Hilbert spectrum plots. 


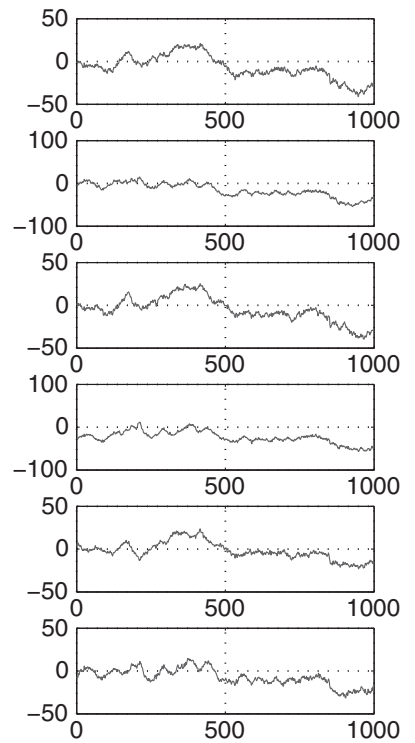

(a) Time-amplitude plot
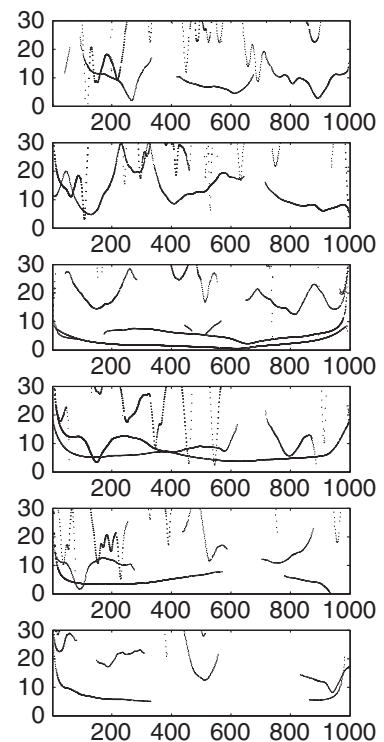

(b)Time-frequency plot

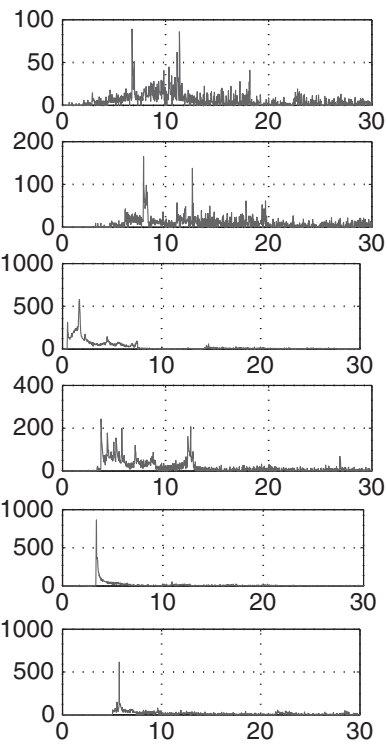

(c) Frequency-amplitude plot

Figure 10.6. Coma to awake.

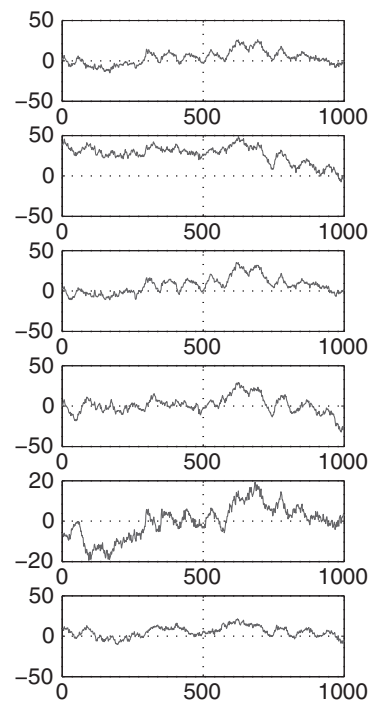

(a) Time-amplitude plot
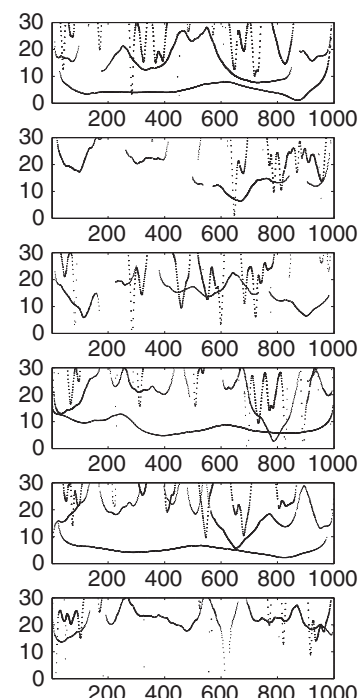

(b) Time-frequency plot

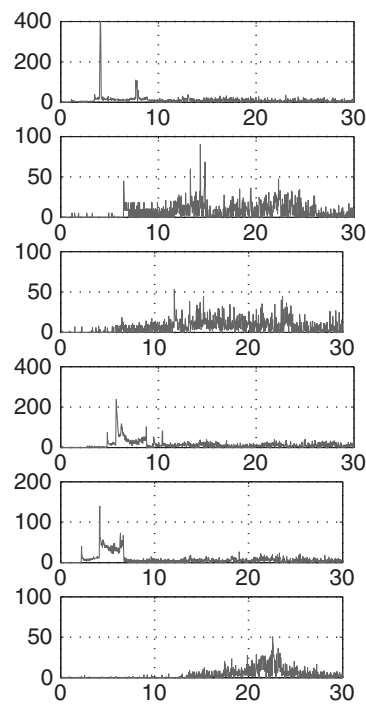

(c) Frequency-amplitude plot

Figure 10.7. Coma to Brain Death. 


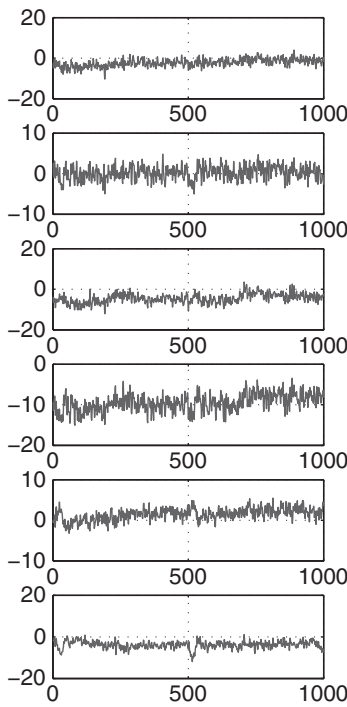

(a) Time-amplitude plot
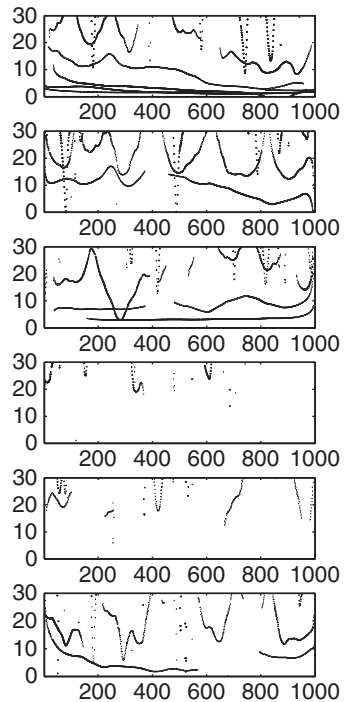

(b) Time-frequency plot
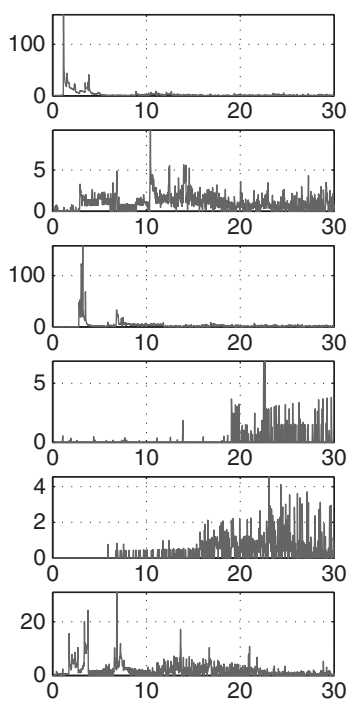

(c) Frequency-amplitude plot

Figure 10.8. Brain Death.

There are several important points to note regarding the features obtained using EMD. First, it is clear that by comparing the frequency-amplitude plots for patient 1 and patient 2, the brain activity within the range of 0 to $13 \mathrm{~Hz}$ is significantly weaker for patient 2. As mentioned earlier, activity within this range is related to the low level and meditative brain activity. It can therefore be suggested that there is little or no activity within this crucial range and that it can be used as an indication of inevitable brain death. Also note that the same observations can be made from the data given in Figure 10.8, patient 2 at a later date. The second point to note is that there exists strong similarity between the spectra obtained from EEG channels of patient 1. For example, compare the frequency-amplitude plots of FP1 and FP2 in Figure 10.6. However, it is clear that this is not the case for patient 2 . It can be thus concluded that strong similarities in the range 0 to $13 \mathrm{~Hz}$ (particularly in channel FP1 and FP2) indicate that the patient will not succumb to brain death.

10.5.4.1 EEG Phase Analysis using EMD. It is clear that if similarities exist between specific frequency ranges ( 8 to $13 \mathrm{~Hz}$ ) among different EEG channels (FP1 and FP2), then this is an indication that the patient will not succumb to brain death. For this reason, it is proposed to extract relevant channel features using EMD and to compare these features using complex extensions of the algorithm.

The EEG data from channel FP1 and FP2 for each patient were decomposed into their IMFs and their instantaneous amplitude and frequencies were calculated. Features within the desired frequency range were retained by [17], fusing instantaneous amplitudes that corresponded to instantaneous frequencies within 8 to $13 \mathrm{~Hz}$. All other 

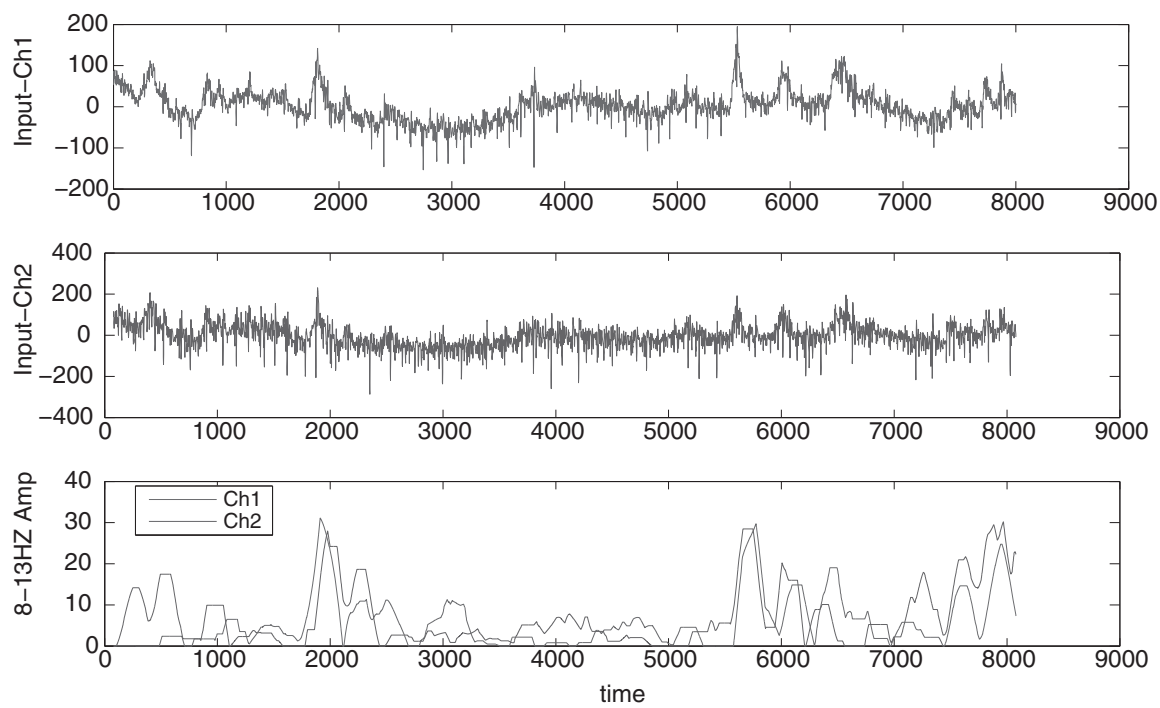

Figure 10.9. Filtered EEG deep coma patient 1 to awake.

instantaneous amplitudes were omitted from the fusion process. The data was subsequently denoised [17] by zeroing the underlying signal if in a certain period, unless the signal continuously has non-zero value in this period. The results of this fusion process for FP1 andFP2 are given in the bottom panel of Figure 10.9 for patient 1. Similarly, the fusion result for FP1 and FP2 for patient 2 is given in the bottom panel of Figure 10.10
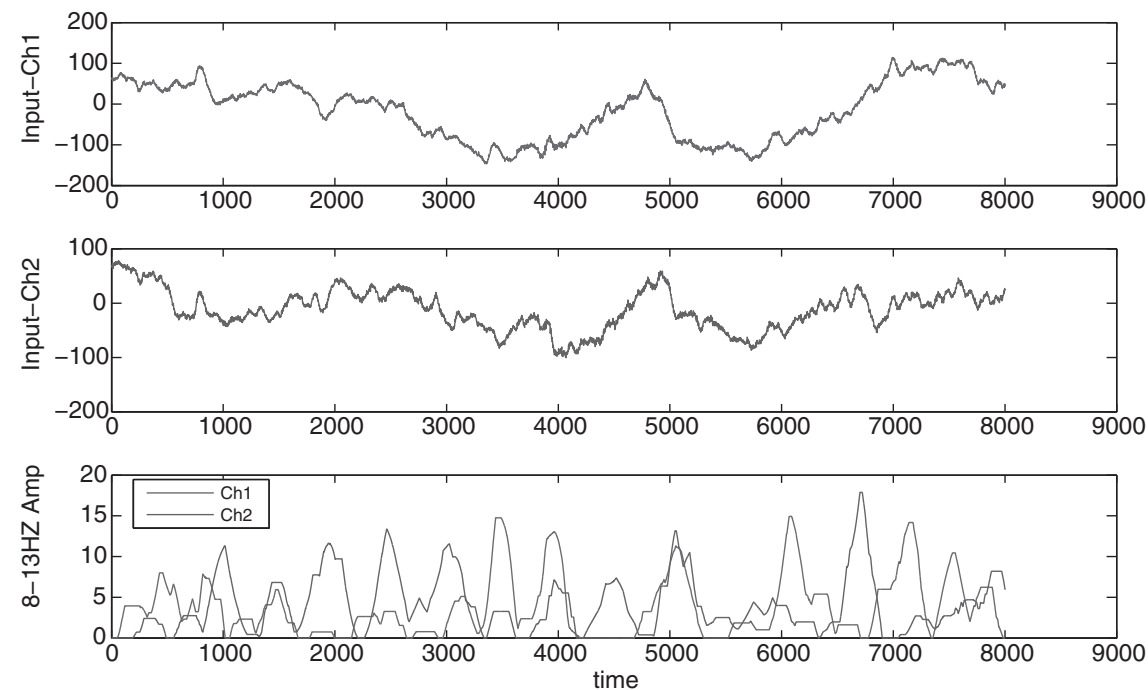

Figure 10.10. Filtered EEG deep coma patient 2 to death. 
as $\mathrm{FP}^{\prime}$ and $\mathrm{FP} 2$, respectively. Note that among the features extracted for patient 1 , there is much synchronization between the extrema. This is not the case for patient 2.

Complex extensions of EMD were then applied to the features so as to highlight any synchronizations that were present. By decomposing the complex signal $\left(\mathrm{FP} 1^{\prime}+j \mathrm{FP} 2^{\prime}\right)$ using rotation-invariant EMD, it was possible to monitor the phase relationship accurately between the two channels. Taking the first complex IMF, it can be assumed extracting the high-frequency information, which can suggest the spikes of the original signal. By design, if the variations of two signals are similar, the phase of the complex signal is approximately $\pi / 4$. As is shown in Figure 10.11 , the panel represents the absolutely value of the first complex IMF. The amplitude peaks when FP1' and FP2' both have a peak. The third plot in Figure 10.11 is a digitalized phase information. If the phase of the first complex IMF is near to $\pi / 4$, the value was set to one; otherwise, the value was set to zero. Then the extracted phase and amplitude information was combined together by multiplying the absolute value of the first IMF with the digitalized phase information. The combined amplitude-phase information can be seen from the plot at the bottom of Figure 10.11. This is regarded as an index for detecting the amplitude-phase similarity of the two signals. The result of the same method applied to the filtered signals $\mathrm{FP}^{\prime}{ }^{\prime}$ and FP2' is shown at the bottom of Figure 10.12.

By comparing the index derived from the coma signals of patient 1 and patient 2, it can be seen that patient 1 is likely to recover. Note that the interval in the digitalized phase information of patient 1 is longer than the interval in the digitalized phase information of patient 2 . This can suggest a quicker phase change in patient 2 . Therefore, the patient should be treated by medical care rather than having further diagnosis tests for braindeath.
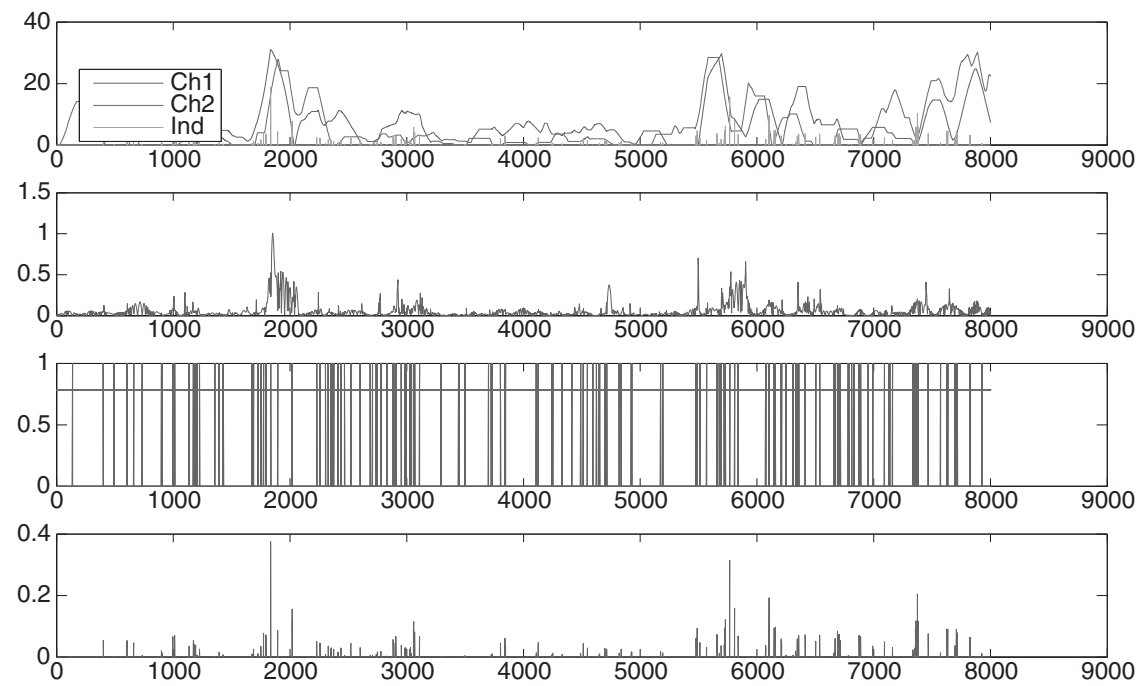

Figure 10.11. Complex EEG analysis deep coma patient 1 leading to awake. 

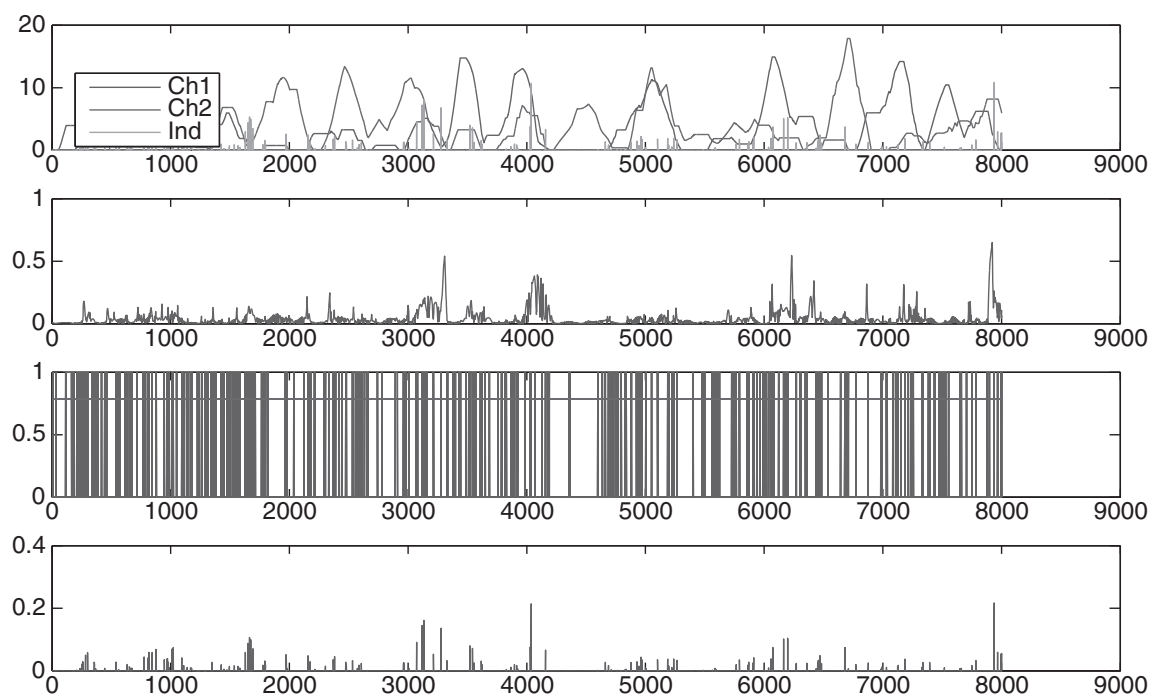

Figure 10.12. Complex EEG analysis deep coma patient 2 leading to brain death.

\subsection{CONCLUSION}

The data fusion via fission concept is introduced in this chapter. Using EMD as the methodology of data fission, then fusing the information of interest, provides a powerful tool for analyzing data. By extending EMD fusion via fission to the complex domain, it can be seen that the methodology is not limited to complex signals, but is also useful when the phase information between two signals is important. It has been illustrated in the area of quasi-brain-death preliminary diagnosis.

\subsection{REFERENCES}

1. M. U. B. Altaf, T. Gautama, T. Tanaka, D.P. Mandic, "Rotation Invariant Complex Empirical Mode Decomposition." Proc. International Conference on Acoustics, Speech and Signal Processing (ICASSP), 2007, Vol. 3, pp. 1009-1012.

2. H. K. Beecher, "A Definition of Irreversible Coma: Report of the Ad Hoc Committee of the Harvard Medical School to Examine the Definition of Brain Death," JAMA: Journal of the American Medical Association, 205: 337-340, 1968.

3. J. Cao, "Analysis of the Quasi-Brain-Death EEG Data Based on a Robust ICA Approach," LNAI 4253, Part III: 1240-1247, KES 2006.

4. G. E. Chatrian, E. Lettich, P. L. Nelson, "Ten Percent Electrode System for Topographic Studies of Spontaneous and Evoked EEG Activity," American Journal of EEG Technology, 25: 83-92, 1985.

5. T. F. Collura, "The Five Percent Electrode System for High-Resolution EEG and ERP Measurements," Clinical Neurophysiology, 112: 713-719, 1997. 
6. M. Datig, T. Schlurmann, "Performance and Limitations of the Hilbert-Huang Transformation (HHT) with an Application to Irregular Water Waves," Ocean Engineering, 31: 1783-1834, October 2004.

7. D.F. Group, “Functional Description of the Data Fusion Process," Technical Report, Office of Naval Technology, 1992.

8. S. L. Hahn, Hilbert Transforms in Signal Processing. Artech House, 1996.

9. D. L. Hall, J. Llinas, “An introduction to Multisensor Data Fusion.” Proc. IEEE, 1997, 85 (1): 6-23.

10. N. E. Huang, Z. Shen, S. R. Long, M. L. Wu, H. H. Shih, Z. Quanan, N. C. Yen, C. C. Tung, H. H. Liu, "The Empirical Mode Decomposition and the Hilbert Spectrum for Nonlinear and non-stationary Time Series Analysis," Proc. Royal Society A, 1998, 454: 903-995.

11. H. H. Jasper. "Report of the Committee on Methods of Clinical Examination in electroencephalography," Electroencephalography and Clinical Neurophysiology, (10): 370-375, 1958.

12. D. Looney, D. P. Danilo, “A Machine Learning Enhanced Empirical Mode Decomposition (Accepted).” Proc. IEEE Int. Conf. on Acoustics, Speech, and Signal Processing (ICASSP'O8), 2008 .

13. D. Looney, L. Li, T. Rutkowski, D. P. Mandic, A. Cichocki, "Ocular Artifacts Removal from EEG Using EMD.” First Int. Conf. Cognitive Neurodynamics, Shanghai, China, November 2007.

14. D. P. Mandic, M. Golz, A. Kuh, D. Obradovic, T. Tanaka, Signal Processing Techniques for Knowledge Extraction and Information Fusion. Springer, 2008.

15. D. P. Mandic, G. Souretis, W. Leong, D. Looney, M. Hulle, T. Tanaka, Signal Process ing Techniques for Knowledge Extraction and Information Fusion. Springer, 2007.

16. A. Paolin, A. Manuali, F. Di Paola, F. Boccaletto, P. Caputo, R. Zanata, G. P. Bardin, G. Simini, "Reliability in diagnosis of brain death," Intensive Care Med, 21: 657-662, 1995.

17. Y. Saito, T. Tanaka, J. Cao, D. P. Danilo, "Quasi-Brain-Death EEG Data Ayalysis by Empirical Mode Decomposition." Proc. ICCN 2007.

18. G. Souretis, D. P. Mandic, M. Griselli, T. Tanaka, M. M. Van Hulle, "Blood Volume Signal Analysis with Empirical Mode Decomposition." Proc. 15th International DSP Conference, 2007, pp. 147-150.

19. T. Tanaka, D. P. Mandic, "Complex Empirical Mode Decomposition," IEEE Signal Processing Letters, 14(2): 101-104, February 2007.

20. J. W. Tukey, Exploratory Data Analysis. Reading, MA: Addison-Wesley, 1977.

21. L. Wald, "Some Terms of Reference in Data Fusion." IEEE Trans. Geosciences and Remote Sensing, 37(3): 1190-1193, 1999.

22. E. Waltz, J. Llinas, Multisensor Data Fusion. Artech House, 1990.

23. R. R. Zhang, S. Ma, E. Safak, S. Hartzell, "Hilbert-Huang Transform Analysis of Dynamic and Earthquake Motion Recordings. J. Engineering Mechanics, 129: 861-875, 2003. 